\title{
TRANSIENT DYNAMICS AND ASYMPTOTIC POPULATIONS IN A DRIVEN METASTABLE QUANTUM SYSTEM*
}

\author{
L. Magazzì ${ }^{\dagger}$, D. Valenti ${ }^{\ddagger}$, P. Caldara, A. La Cognata \\ B. SPAGNOLO ${ }^{\S}$
}

Group of Interdisciplinary Physics, Dipartimento di Fisica e Chimica Università di Palermo and CNISM — Unità di Palermo

Viale delle Scienze 18, 90128 Palermo, Italy

\section{G. FALCI}

Dipartimento di Fisica e Astronomia, Università di Catania Via Santa Sofia 64, 95123 Catania, Italy

\section{(Received April 11, 2013)}

The transient dynamics of a periodically driven metastable quantum system, interacting with a heat bath, is investigated. The time evolution of the populations, within the framework of the Feynman-Vernon influence functional and in the discrete variable representation, is analyzed by varying the parameters of the external driving. The results display strong non-monotonic behaviour of the populations with respect to the driving frequency.

DOI:10.5506/APhysPolB.44.1185

PACS numbers: 03.65.Aa, 03.65.Yz, 05.40.-a

\section{Introduction}

A variety of physical systems in which quantum tunneling plays an important role, of interest especially in the field of condensed matter, can be modeled as quantum bistable systems in the presence of a noisy environment. In particular, the relaxation from a metastable state under the

* Presented at the XXV Marian Smoluchowski Symposium on Statistical Physics, "Fluctuation Relations in Nonequilibrium Regime", Kraków, Poland, September 10-13, 2012.

$\dagger$ luca.magazzu@.unipa.it

$\ddagger$ davide.valenti@.unipa.it

$\S$ bernardo.spagnolo@.unipa.it 
influence of a dissipative environment constitutes an interesting application of the theory of this class of open quantum systems [1-3] to devices for quantum computation such as SQUIDs, multilevels nanodevices and others [4-12]. We consider a periodically driven quantum system, characterized by a metastable state, in interaction with a thermal bath described by the Caldeira-Leggett model [13]. We study the transient dynamics as well as the asymptotic values of the populations in the Discrete Variable Representation (DVR), in the strong coupling regime. This is done by following the analytical approach (given in [14]) based on the spatial discretization of a double dissipative path integral for the particle's density matrix elements, containing the Feynman-Vernon influence functional [15]. The populations dynamics is obtained for two values of coupling strength and by varying the parameters of the periodical driving.

\section{The model}

A quantum particle is subject to a bistable potential with an asymmetry term. A periodical driving of amplitude $A$ and angular frequency $\Omega$ is also applied. The particle, which we identify with $\mathrm{S}$, the system, has mass $M$ and position operator $\hat{q}$. It evolves, starting from $t=t_{0}$, under the influence of a heat bath, the environment $\mathrm{B}$, modeled as a reservoir of $N$ mutually independent quantum harmonic oscillators, with frequencies $\omega_{j}$ and position degrees of freedom $\hat{x_{j}}$, linearly interacting with the system (Caldeira-Leggett model [13]). The total Hamiltonian is

$$
\begin{aligned}
\hat{H}(t) & =\hat{H}_{\mathrm{S}}(t)+\hat{H}_{\mathrm{B}+\mathrm{SB}} \\
\hat{H}_{\mathrm{S}}(t) & =\frac{\hat{p}^{2}}{2 M}+\hat{V}_{0}(\hat{q})-\hat{q} A \sin \Omega t \\
\hat{V}_{0}(\hat{q}) & =\frac{M^{2} \omega_{0}^{4}}{64 \Delta U} \hat{q}^{4}-\frac{M \omega_{0}^{2}}{4} \hat{q}^{2}-\epsilon \hat{q}, \\
\hat{H}_{\mathrm{B}+\mathrm{SB}} & =\sum_{j=1}^{N} \frac{1}{2}\left[\frac{\hat{p}_{j}^{2}}{m_{j}}+m_{j} \omega_{j}^{2}\left(\hat{x}_{j}-\frac{c_{j}}{m_{j} \omega_{j}^{2}} \hat{q}\right)^{2}\right] .
\end{aligned}
$$

The potential and the first eight energy levels are shown in Fig. 1.

It is assumed that the particle-bath total density matrix $\chi(t)$ is initially in the factorized form $\chi\left(t_{0}\right)=\rho\left(t_{0}\right) \otimes \rho_{\mathrm{Th}}^{\mathrm{B}}$, where $\rho(t)$ denotes the particle's density matrix and $\rho_{\text {Th }}^{\mathrm{B}}$ represents the thermal state of the bath. Once specified the Hamiltonian, the particle's density matrix, obtained by performing the partial trace over the bath's degrees of freedom on $\chi(t)$, evolves according to

$$
\rho\left(q_{f}, q_{f}^{\prime}, t\right)=\int d q_{0} \int d q_{0}^{\prime} G\left(q_{f}, q_{f}^{\prime}, t ; q_{0}, q_{0}^{\prime}, t_{0}\right) \rho\left(q_{0}, q_{0}^{\prime}, t_{0}\right) .
$$


The propagator $G\left(q_{f}, q_{f}^{\prime}, t ; q_{0}, q_{0}^{\prime}, t_{0}\right)$ has the form of a double path integral [14] in which the free evolution term, induced by $\hat{H}_{\mathrm{S}}(t)$, and the FeynmanVernon functional $\mathcal{F}_{\mathrm{FV}}\left[q(t), q^{\prime}(t)\right]=e^{-\frac{1}{\hbar} \Phi_{\mathrm{FV}}\left[q(t), q^{\prime}(t)\right]}$ [15], quantifying the influence exerted by the bath, are factorized. $\mathcal{F}_{\mathrm{FV}}$ accounts for the bath's temperature $T$ and its spectral density $J(\omega)$ which, in the limit $N \rightarrow \infty$, is assumed to be Ohmic [2]

$$
J(\omega)=M \gamma \omega \exp \left(-\frac{\omega}{\omega_{\mathrm{c}}}\right) .
$$

The cut-off frequency $\omega_{\mathrm{c}}$ is chosen so as to be the largest frequency compared to $\omega_{0}$ and to the splittings between the energy levels associated to the static potential (see Fig. 1), involved in the dynamic. The damping coefficient $\gamma$ measures the system-bath coupling strength and, in the case of Ohmic bath, is frequency independent.

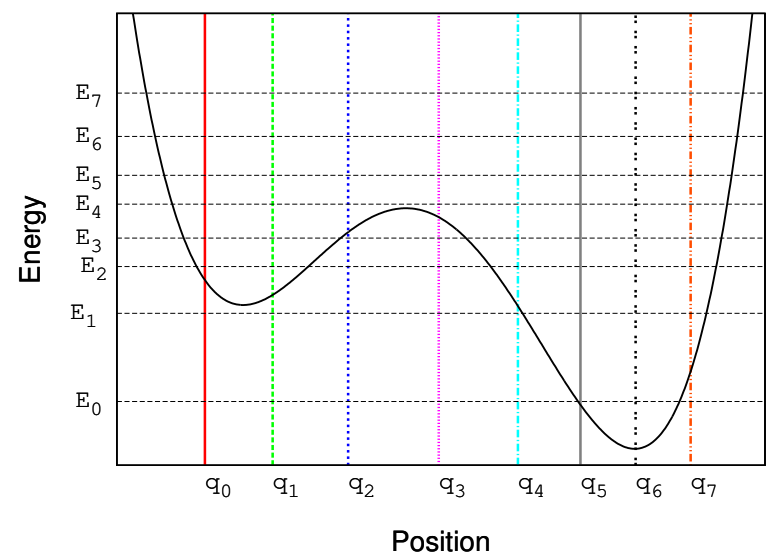

Fig. 1. Static potential, first eight energy levels (horizontal lines) and position eigenvalues (vertical lines) in the DVR. $M=\omega_{0}=\hbar=1, \Delta U=1.8, \epsilon=0.21$.

\section{Analytical approach}

For appropriate values of $\gamma, T$ and of the driving parameters [14], we can describe the dynamics in terms of transitions among the first $M$ unperturbed energy levels $\left\{E_{0}, \ldots, E_{M-1}\right\}$, by assuming that the system is not going to be excited to higher energies (in this work $M$ is set to 8). By means of a suitable transformation on the basis set $\left\{\left|E_{i}\right\rangle\right\}$, we obtain the basis set of eigenfunctions of the $\hat{q}$ operator with eigenvalues $\left\{q_{0}, \ldots, q_{M-1}\right\}$ providing the Discrete Variable Representation (DVR) [16]. 
In the DVR, we represent the dynamics of the system's density matrix as a sequence of transitions among the discrete set of spatial positions $\left\{q_{\mu}\right\}$. As a consequence, by making the identification $\rho\left(q_{\mu}, q_{\nu}, t\right) \equiv \rho_{\mu \nu}(t)$, if we are interested in the time evolution of a diagonal density matrix element $\left(q(t)=q^{\prime}(t)\right)$ Eq. (5) turns into

$$
\rho_{\mu \mu}(t)=\sum_{\mu_{0}, \nu_{0}=0}^{M-1} G_{\mu \mu, \mu_{0}, \nu_{0}}\left(t, t_{0}\right) \rho_{\mu_{0}, \nu_{0}}\left(t_{0}\right) .
$$

Here, $G_{\mu \mu, \mu_{0}, \nu_{0}}\left(t, t_{0}\right)$ is the discretized version of the double dissipative path integral in Eq. (5). The phase of the Feynman-Vernon influence for paths with transitions at intermediate times $\left\{t_{i}\right\}$, is

$$
\Phi_{\mathrm{FV}}=-\sum_{i=1}^{m} \sum_{l=0}^{i-1} \xi_{i} C^{\prime}\left(t_{i}-t_{l}\right) \xi_{l}-i \sum_{i=1}^{m} \sum_{l=0}^{i-1} \xi_{i} C^{\prime \prime}\left(t_{i}-t_{l}\right) \chi_{l}
$$

where $C^{\prime}(\tau)$ and $C^{\prime \prime}(\tau)$ are real and imaginary part of the bath correlation function, respectively. The quantities $\xi_{i}=q_{\mu}\left(t_{i}\right)-q_{\nu}\left(t_{i}\right)-\left[q_{\mu}\left(t_{i-1}\right)-q_{\nu}\left(t_{i-1}\right)\right]$ and $\chi_{i}=q_{\mu}\left(t_{i}\right)+q_{\nu}\left(t_{i}\right)-\left[q_{\mu}\left(t_{i-1}\right)+q_{\nu}\left(t_{i-1}\right)\right]$, are named charges. The $\xi$-charges sum up to zero for every piece of path connecting two diagonal elements $\left(q_{\mu}\left(t_{i}\right)=q_{\nu}\left(t_{i}\right)\right)$. As a consequence, inside a path, we can make the approximation of neglecting all the interactions between different nondiagonal pieces connecting diagonal elements, thus realizing the generalized non-interacting cluster approximation (gNICA) [14] (a cluster is the time interval during which $q_{\mu} \neq q_{\nu}$ ). Moreover, if we represent the couples of position eigenvalues $\left\{q_{\mu}, q_{\nu}\right\}$ (defining $\rho_{\mu \nu}\left(t^{\prime}\right)$ at an intermediate time $t^{\prime}$ ) in a two dimensional spatial grid, we take into account only paths made up by single excursions to off- diagonal states, followed by the return to diagonal states. The effect is of drastically reducing the number of terms in the sum over the paths.

By the use of Laplace transforms, a master equation for the populations $\rho_{\mu \mu}(t)$ is obtained. In the Markovian limit and under the hypotesis that the system is initially prepared in a diagonal state $\rho_{\mu_{0} \mu_{0}}\left(t_{0}\right)$ (in the DVR this means a localized state), it reads

$$
\dot{\rho}_{\mu \mu}(t)=\sum_{\nu=0}^{M-1} \Gamma_{\mu \nu}(t) \rho_{\nu \nu}(t) .
$$

The time dependence in the rate matrix coefficients $\Gamma_{\mu \nu}(t)$ is due to the presence of the time dependent driving force. However, for high enough 
driving frequencies $\Omega$ [14], we can make an average over a period $\frac{2 \pi}{\Omega}$ and solve the master equation with averaged coefficients

$$
\Gamma_{\mu \nu}(t) \rightarrow \Gamma_{\mu \nu}^{\mathrm{av}}=\frac{\Omega}{2 \pi} \int_{0}^{\frac{2 \pi}{\Omega}} d t \Gamma_{\mu \nu}(t) .
$$

Equation (9), with the averaged rate matrix (Eq. (10)), has solution in the form

$$
\rho_{\mu \mu}(t)=\sum_{\lambda, \nu=0}^{L-1} S_{\mu \lambda}\left(S^{-1}\right)_{\lambda \nu} e^{\Lambda_{\lambda} t} \rho_{\nu \nu}\left(t_{0}\right),
$$

where $\Lambda_{\lambda}$ are the eigenvalues of the rate matrix $\Gamma^{\mathrm{av}}$. By the conservation of the probability, one can infer that one of them, $\Lambda_{0}$, is zero and fixes the asymptotic value $\rho_{\mu \mu}^{\infty}=\sum_{\nu=0}^{L-1} S_{\mu 0}\left(S^{-1}\right)_{0 \nu} \rho_{\nu \nu}\left(t_{0}\right)$ of the population $\rho_{\mu \mu}(t)$.

\section{Results}

We set the number of energy levels to $M=8$, and the temperature to the value of $T=0.15$, corresponding to the intermediate temperature regime, compared with the barrier height of the potential used (see Fig 1). In this work, $M=\omega_{0}=\hbar=k_{\mathrm{B}}=1$. Every result is obtained for $\gamma=0.1$ and $\gamma=0.3$, giving the strong coupling regime. In fact, the interaction with the bath cannot be regarded as a small perturbation.

\subsection{Dynamics}

The transient dynamics of the populations in the DVR, given by Eq. (11), is shown for $\gamma=0.1$ in Fig. 2 and for $\gamma=0.3$ in Fig. 3. In both cases, we compare (a) the non-driven evolution and (b) the evolution in the presence of driving with frequency $\Omega$ comparable to the average energy difference between the DVR states in the two wells. We have chosen as initial condition for the particle $\rho(0)=\left|q_{3}\right\rangle\left\langle q_{3}\right|$, corresponding to a DVR state with position eigenvalue in the region of instability of the potential (see Fig 1). The evolutions starts at $t_{0}=0$.

For both the values of $\gamma$ considered, the transient dynamics displays a peak in the population located in the metastable well. We notice that the duration of the transient depends on the coupling strength $\gamma$, the transient lasting more for $\gamma=0.3$ than for $\gamma=0.1$. In both cases, the presence of the driving has the effect of accelerating the dynamics.

After the transient, in the absence of driving, the particle relaxes completely towards the lower well, while in the driven regime, the metastable well remains populated. The asymptotic populations of the metastable DVR 
states, that is $\left|q_{0}\right\rangle,\left|q_{1}\right\rangle$ and $\left|q_{2}\right\rangle$ (see Fig. 1), diminish as we increase the coupling strength $\gamma$. This is because the bath forces the relaxation to the lower well (see the next section).
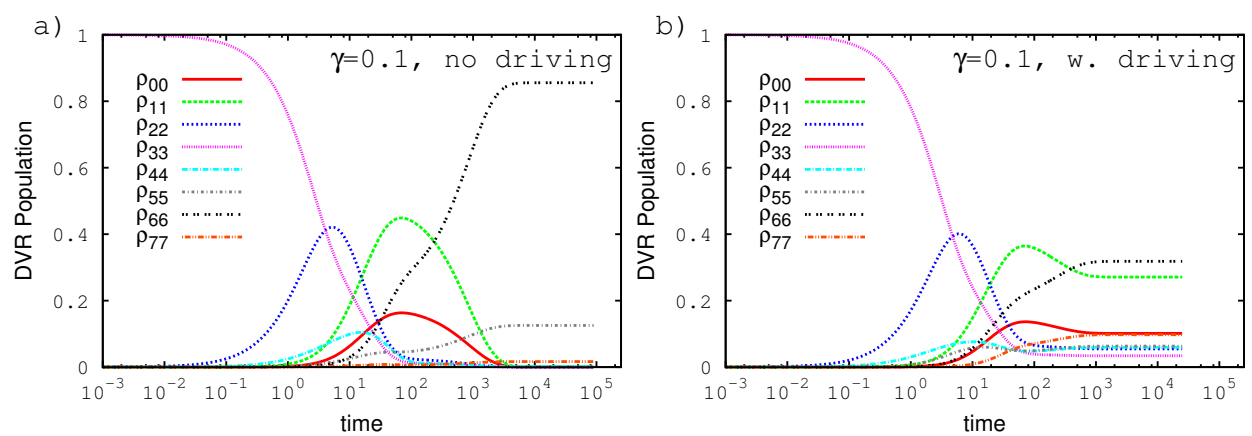

Fig. 2. Dynamics of the DVR populations for $\gamma=0.1$ (a) in the absence of external driving $(A=0)$ and (b) with the external driving $(A=0.25$ and $\Omega=0.7)$. The bath temperature is $T=0.15$. The potential parameters are: $\omega_{0}=\hbar=1$, $\Delta U=1.8, \epsilon=0.21$, as in Fig. 1 .
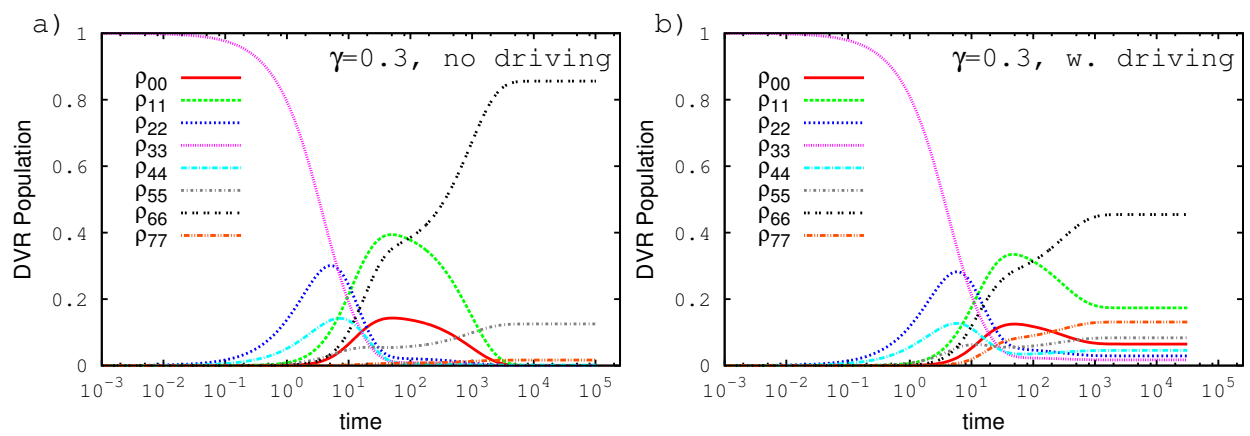

Fig. 3. Dynamics of the DVR populations for $\gamma=0.3$ (a) in the absence of external driving $(A=0)$ and (b) with the external driving $(A=0.25$ and $\Omega=0.7)$. The bath temperature is $T=0.15$. The potential is the same as in Fig. 2 .

\subsection{Asymptotic populations}

We define the asymptotic population of the metastable well as $\rho_{\mathrm{Left}}^{\infty}=$ $\sum_{\mu=0}^{2} \rho_{\mu \mu}^{\infty}$, that is the sum of the asymptotic populations of the three DVR states with positions in the left well in Fig. 1. Two graphs are shown in which this quantity is plotted as a function of both the amplitude $A$ and the frequency $\Omega$ of the external driving, corresponding to the two coupling regimes considered (Fig. 4). In both cases, there is a strong non-monotonicity as the frequency is varied. 



Fig. 4. Asymptotic left well population as a function of both amplitude $A$ and frequency $\Omega$ of the external driving, for two values of the coupling strength: (a) $\gamma=0.1$ and (b) $\gamma=0.3$. The bath temperature is $T=0.1$.

For $\gamma=0.1$, we observe the presence of various resonance peaks associated with the level structure of the static potential used. In particular, at $\Omega \simeq 0.7$ the metastable well asymptotic population is noticeably different from zero even for moderate values of the amplitude $A$. As the frequency is increased beyond $\simeq 1.6$, the curve tends smoothly to the non-driven population values.

In the $\gamma=0.3$ case, the peaks in the high frequency domain disappear and the asymptotic population has the same values as in the non-driven case $(\simeq 0)$. On the other hand, in the lower frequency part of the range considered here, the peak at $\Omega \simeq 0.7$ shows a robustness against the tendency to the complete relaxation induced by the strong particle-bath coupling. It is indeed comparable to the corresponding one in the weaker coupling regime. A similar consideration holds for the peak in population at $\Omega \simeq 0.3$.

\section{Conclusions}

The transient dynamics of the quantum particle, initially localized in an unstable position relative to a bistable asymmetric potential, is studied in the presence of environmental noise, introduced by the coupling with a heat bath, and of a periodically varying driving potential. This is done in the strong coupling regime, for two values of the coupling strength. The resulting driven dynamics are compared with the corresponding obtained in the absence of driving.

In both coupling regimes, during the transient, the population of the DVR states belonging to the metastable well reaches a maximum and then goes to zero (complete relaxation) in the non-driven case, while, in the driven case they stabilize on values that depend on the driving parameters. The duration of the transient is greater for the higher value of the coupling strength. As the driving frequency is varied, the behaviour of the stationary value of the metastable well population is strongly non-monotonic, with 
peaks in correspondence of resonant frequencies as determined by the static potential. The peaks in the high driving frequency domain are removed as we pass from the lower to the higher value of the coupling strength.

This work was partially supported by MIUR, CNISM, and by MIUR through Grant. No. PON02 00355 3391233, ENERGETIC.

\section{REFERENCES}

[1] H.P. Breuer, F. Petruccione, The Theory of Open Quantum Systems, Claredon Press, Oxford 2006.

[2] U. Weiss, Quantum Dissipative Systems, 3rd ed., World Scientific, Singapore 2008.

[3] M. Grifoni, P. Hänggi, Phys. Rep. 304, 229 (1998).

[4] J.Q. You, F. Nori, Nature 474, 589 (2011).

[5] J.R. Friedman et al., Nature 406, 43 (2000).

[6] J.M. Martinis, S. Nam, J. Aumentado, C. Urbina, Phys. Rev. Lett. 89, 117901 (2002).

[7] P. Caldara et al., Int. J. Quant. Inf. 9, 119 (2011).

[8] A. La Cognata et al., Int. J. Quant. Inf. 9, 1 (2011).

[9] J. Siewert, T. Brandes, G. Falci, Phys. Rev. B79, 024504 (2009).

[10] F. Chiarello et al., New J. Phys. 14, 023031 (2012).

[11] B. Spagnolo et al., Acta Phys. Pol. B 43, 1169 (2012).

[12] G. Falci et al., Phys. Scr. T151, 014020 (2012).

[13] A.O. Caldeira, A.L. Leggett, Phys. Rev. Lett. 46, 211 (1981).

[14] M. Thorwart, M. Grifoni, P. Hänggi, Ann. Phys. 293, 15 (2001).

[15] R.P. Feynman, F.L. Vernon, Ann. Phys. 24, 118 (1963).

[16] D.O. Harris, G.G. Engerholm, W.D. Gwinn, J. Chem. Phys. 43, 1515 (1965). 